J. Clin. Chem. Clin. Biochem.

Vol. 15, 1977, pp. 687-690

\title{
Isolierung einiger Metaboliten des Clofedanol aus menschlichem Harn und ihre Identifizierung
}

\author{
Von G. Eckhardt
}

Institut für Organische Chemie und Biochemie der Universität Bonn,

and

\section{S. Goenechea und G. Tondorf}

Institut für Gerichtliche Medizin der Universität Bonn

(Eingegangen am 16. März/7. Juli 1977)

Zusammenfassung: Nach Einnahme von Clofedanol (1-(o-Chlorphenyl)-1-phenyl-3-dimethylaminopropan-1-ol) wurden im menschlichen Harn 10 Ausscheidungsprodukte identifiziert. Fünf von ihnen wurden bei pH 1-2 mit Chloroform extrahiert; es handelt sich um $o$-(Chlorphenyl)-phenylmethan (I), Benzophenon (II), $o$-Chlorbenzophenon (III), Benzhydrol (IV) und $o$-(Chlorphenyl)- $\beta$-phenylacrolein (V). Bei pH 13-14 wurden mit Chloroform zwei Verbindungen extrahiert: unverändertes Clofedanol und 1-(o-Chlorphenyl)-1-phenyl-3-dimethylaminopropen-1 (VI). Im Harn konnte ferner N, N-Dimethylaminoessigsäure (VII) identifiziert werden. Clofedanol und Metabolit IV wurden frei und nach Spaltung mit $\beta$-Glucuronidase (VIII und IX) nachgewiesen.

\section{Isolation and identification of some metabolites of Clofedanol from human urine}

Summary: 10 excretory products of Clofedanol (1-(o-chlorphenyl)-1-phenyl-3-dimethylaminopropanol) were identified in human urine. The following five products were extracted with chloroform at $\mathrm{pH} 1-2: 0$-(chlorphenyl)phenylmethane (I), benzophenone (II), $o$-chlorbenzophenone (III), benzhydrol (IV), and $o$-(chlorphenyl)- $\beta$-phenylacrolein (V). Two compounds were extracted with chloroform at $\mathrm{pH}$ 13-14: unchanged Clofedanol and 1-(o-chlorphenyl)-1-phenyl-3-dimethylaminopropene-1 (VI). N,N-dimethylamino-acetic acid (VII) was also identified in urine. Clofedanol and metabolite IV were present as free compounds, and they were also found after hydrolysis of metabolites VIII and IX with $\beta$-glucuronidase.

\section{Einführung}

Clofedanöl (1-(o-Chlorphenyl)-1-phenyl-3-dimethylaminopropan-1-ol-hydrochlorid) wird in der Medizin als hustenhemmender Stoff angewandt. Uber die Biotransformation dieser Substanz ist bisher nichts bekannt. Bei unseren Untersuchungen wurden im menschlichen Harn außer dem unveränderten Clofedanol und seinem Glucuronid acht Ausscheidungsprodukte identifiziert.

\section{Arbeitsmethodik}

Eine gesunde, freiwillige männliche Versuchsperson (26 Jahre alt) nahm in mehreren Żeiträumen insgesamt 60 Tage lang viermal täglich 40 mg Clofedanol-Base in magenlöslichen Gelatinkapseln ein. Der Harn wurde gesammelt und im Kühlschrank ohne Zusätze bis zưr Aufbereitung bei $+2-4^{\circ} \mathrm{C}$ aufbewahrt.
Die Extraktion des Harnes erfolgte bei pH 1-2, 13-14 und schließlich bei pH 7 mit Chloroform. Danach wurde der Harn mit $\mathrm{HCl}$ hydrolysiert und erneut wie beschrieben ausgeschüttelt (genaue Vorschrift s. Lc. (1)). Eine von derselben Versuchsperson erhaltene medikamentenfreie Harnprobe wurde in gleicher Weise aufbereitet und untersucht.

Für die Schichtchromatographie wurden Platten von $200 \mathrm{~mm}$ $\times 200 \mathrm{~mm}$ verwendet, die nach dem Standardverfahren von Stahl (2) mit Kieselgel HF $254+366$ (analytische Schichtchromatographie) bzw. $\mathbf{P F}_{254}$ (präparative Schichtchromatographie) beschichtet wurden. Als Fließmittel wurden verwendet:

1. Chloroform/Aceton/Ameisensäure (Volumina $70 \mathrm{ml}+29 \mathrm{ml}$ $+1 \mathrm{ml})$ :

2. Chloroform/Aceton (Volumina $70 \mathrm{ml}+30 \mathrm{ml}$ )

3. Benzol/Chloroform (Volumina $90 \mathrm{ml}+10 \mathrm{ml}$ )

4. Chloroform/Benzol/Ammoniak-Lösung ( $250 \mathrm{~g} / \mathrm{kg}$ ) (Volumina $40 \mathrm{ml}+40 \mathrm{ml}+20 \mathrm{ml}$ ) 
Als Nachweisreagenzien wurden benutzt:

A) Kaliumjodoplatinat (3)

B) Dragendorff-Reagenz modifiziert nach Thies \& Reuther (Vorratslösung) (3).

C) Reagenz B und Nachbehandlung mit Eisen(III)-chlorid/ $\mathrm{H}_{2} \mathrm{SO}_{4}$ : Eisen(III)-chlorid, $50 \mathrm{~g} / 1$ Wasser (10 Volumina) mit $\mathrm{H}_{2} \mathrm{SO}_{4}, 970 \mathrm{~g} / \mathrm{kg}$ (1 Volumen) versetzen.

Für die präparative Schichtchromatographie wurde - ausgenommen bei Fließmittel 4 - immer die Technik der Mehrfachentwicklung angewandt. Die Auswertung der Chromatogramme und die Isolierung der Ausscheidungsprodukte erfolgte wie in 1. c. (1) angegeben.

Die Untersuchung auf Konjugate erfolgte wie bei l.c. (1). Die enzymatische Spaltung wurde hier allerdings mit $\beta$-Glucuronidase bei $\mathrm{pH} 4,5$ durchgeführt.

Zur Isolierung von $N, N$-Dimethylaminoessigsäure wurden 5 Liter Harn nach Medikamenteneinnahme verwendet. Sie erfolgte im wesentlichen nach dem im Handbuch von Stahl (2) (Kapitel V.: Aminosäuren und Derivate, Abschnitt III. 3, S. 696-749) vorgeschlagenen Verfahren, nur bei der Abtrennung von Eiweiß und Polysacchariden und bei der Entfernung der Lipide wurde nach der Methode von Awapara (4) gearbeitet.

Für die Versuche mit Leberhomogenaten wurden die benötigten Lebern (durchschnittliches Gewicht $10 \mathrm{~g}$ ) weißen Wistar-Ratten (Gewicht 300-450 g) entnommen und sofort eingefroren. $40 \mathrm{~g}$ der harten, noch gefrorenen Lebern wurden vor Versuchsbeginn zuerst in einem Porzellanmörser grob zerkleinert und nach Zugabe von physiologischer NaCl-Lösung homogenisiert. Dem Leberhomogenat wurden $60 \mathrm{mg}$ Clofedanol-Base bzw. $62 \mathrm{mg}$ des synthetisierten Metabolit VI zugesetzt. Die Inkubationszeit betrug 5 Stunden (Temperatur $36^{\circ} \mathrm{C}$ ). Nach Ablauf der Reaktionszeit wurde das Gemisch mit $\mathrm{HCl}(100 \mathrm{~g} / \mathrm{kg})$ auf pH 1 eingestellt, kräftig durchmischt und filtriert. Das Filtrat wurde mit festem $\mathrm{NaCl}$ gesättigt und erst mit $\ddot{A}$ ther, dann mit Chloroform extrahiert; danach wurde wie beschrieben bei $\mathrm{pH}$ 13-14 erneut extrahiert. Die bei saurer Reaktion erhaltenen organischen Extrakte wurden vereinigt, über Natriumsulfat getrocknet, filtriert und das Filtrat wurde bei vermindertem Druck im Rotationsverdampfer eingeengt. Genauso wurde mit den bei pH 13-14 erhaltenen Extrakten verfahren.

\section{Geräte}

Die UV-Spektren wurdeñ mit einem Unicam-Leitz-Spektrophotometer SP 800 B aufgenommen. Die Aufnahme der IRSpektren erfolgte mit einem Perkin-Elmer-Spektrophotometer 221 mit Gitter-Prisma-Austausch-Einheit - wenn nicht anders angegeben - in $\mathrm{KBr}$ unter Standardbedingungen. Die Aufnahme der Massenspektren erfolgte durch direkte Einfuihrung in das MS-9 (AEI) bei einer Ionenquellentemperatur von $150-190^{\circ} \mathrm{C}$, einem Emmissionsstrom von $100 \mu \mathrm{A}$ und Ionisierungsenergien von 12 und $70 \mathrm{eV}$.

\section{Ergebnisse und Diskussion}

In Abbildung 1 sind die gefundenen Ausscheidungsproduktè des Clofedanol angegeben.

Vò der salżsauren Hydrolyse wurden 7 Ausscheidungsprodukte extrahiert; fünf von ihnen - Verbindungen I, II, III, IV und V - wurden aus dem angesäuerten Harn, die anderen zwei bei alkalischer Reaktion ausgeschüttelt. Bei einer der basischen Substanzen handelt es sich - wie der dünnschichtchromatographische Vergleich sowie der Vergleich der UV-, IR- und Massenspektren mit denen des authentischen Materials ergab - um Clofedanol; dieses und die andere basische Verbindung (Metabolit VI) waren durch ihre charakteristische Färbung mit Reagenz A (violett) und B (orange) auf dem Chromatogramm gut erkennbar. Die in dem sauren Auszug gefundenen Substanzen konnten dagegen nur durch die Fluoreszenzlöschung im kurzwelligen UV-Licht lokali-<smiles>COC(=O)CCC(O)(c1ccccc1Cl)c1ccccc1Cl</smiles>

Abb. 1. Abbauschema des Clofedanol 
siert werden. Nach salzsaurer Hydrolyse und nach enzymatischer Spaltung wurden zwei Substanzen nachgewiesen: Metabolit IV in dem sauren und Clofedanol in dem alkalischen Auszug.

Für die Chromatographie der Metaboliten I-V wurde das Fließmittel 1 benutzt; die Metaboliten II und III ließen sich am besten mit Fließmittel 3 trennen. Clofedanol und Metabolit VI wurden mit dem Fließmittel 2 chromatographiert. Metabolit I wurde aufgrund seines UV- (Absorptionsmaxima bei 254, 262 und $269 \mathrm{~nm}$ ) und Massenspektrums als o-(Chlorphenyl)phenylmethan erkannt. Die wichtigste Spaltung beim Elektronenstoß-induzierten Zerfall ist die Eliminierung eines $\mathrm{Cl}$-Atoms aus dem Molekülion $\mathrm{C}_{13} \mathrm{H}_{11} \mathrm{Cl}^{+}$. $(\mathrm{m} / \mathrm{e}$ 202; 100\%) zu dem Fragment m/e 167 (52\%), das anschließend ein bzw. zwei H-Atome verliert, zu m/e $166(48 \%)$ und $\mathrm{m} / \mathrm{e} 165$ (54\%). Durch hochauflösende Massenspektrometrie und durch den dünnschichtchromatographischen und absorptionspektroskopischen Vergleich mit den authentischen Verbindungen wurde Metabolit II als Benzophenon, Metabolit III als $o$-Chlorbenzophenon und Metabolit IV als Benzhydrol identifiziert.

Das bei $70 \mathrm{eV}$ aufgenommene Massenspektrum von Metabolit $\mathrm{V}$ zeigt bei $\mathrm{m} / \mathrm{e} 242$ einen wenig intensiven Peak (7\%) für das Molekülion, dessen Elementarzusammensetzung durch Hochauflösung $\mathrm{zu} \mathrm{C}_{15} \mathrm{H}_{11} \mathrm{ClO}$ bestimmt wurde. Durch Abspaltung eines H-Atoms wird das Ion m/e 241 (9\%), durch Abspaltung eines Cl-Atoms der Basispeak bei m/e 207 (98\%) gebildet. Diese Fragmentierungsreaktionen legen nahe, daß es sich bei Metabolit V um $\beta$-(o-Chlorphenyl)- $\beta$-phenylacrolein handelt $(5,6,7)$. Mit dieser Struktur im Einklang ist das IR-Spektrum, welches bei $1665 \mathrm{~cm}^{-1}$ eine intensive Bande zeigt, die einer $\alpha, \beta$-ungesättigten Carbonylgruppe zugeordnet werden kann. Metabolit V ergibt in Methanol ein Absorptionsmaximum bei $312 \mathrm{~nm}$.

Die durch hochauflösende Massenspektroskopie zu $\mathrm{C}_{17} \mathrm{H}_{16} \mathrm{NCl}$ bestimmte Elementarzusammensetzung des Molekülions weist Metabolit VI als Dehydratisierungsprodukt der Clofedanol-Base aus. Das IR-Spektrum - aufgenommen zwischen NaCl-Platten - zeigt bei 2765 und $2815 \mathrm{~cm}^{-1}$ die typischen Banden für eine - $\mathrm{N}\left(\mathrm{CH}_{3}\right)_{2}$-Gruppe auf, dazu Absorptionen für die Aromaten $\left(3080,3055\right.$ und $3022 \mathrm{~cm}^{-1}, \vartheta-\mathrm{CH} ; 1590$, 1560 und $1490, C=C)$. Eine mittelstarke Bande bei $820 \mathrm{~cm}^{-1}$, die im Spektrum von Clofedanol-Base fehlt, kann der $\gamma$-CH-Schwingung der trisubstituierten Doppelbindung zugeordnet werden. Somit kommt Metabolit VI die angegebene Struktur zu. Damit im Einklang ist die geringe Intensität des Fragments $\mathrm{CH}_{2}=\mathrm{N}^{+}\left(\mathrm{CH}_{3}\right)_{2}$ (m/e 58) im Massenspektrum von VI (38\%) im Ver: gleich zu dem im Spektrum von Clofedanol-Base (100\%), da im Fall von Metabolit VI déssen Bịldung die Spaltung einner Vịnylbindung erfordert.

Durch Umsetzung von Clofedanol-Base mit $\mathrm{H}_{3} \mathrm{PO}_{4}$ wurde 1-(o-Chlorphenyl)-1-phenyl-3-dimethylamino- propen-(1) erhalten. Diese Verbindung erwies sich dünnschichtchromatographisch, UV-, IR- und massenspektrometrisch als identisch mit Metabolit VI. Das in Methanol aufgenommene UV-Spektrum von VI zeigt ein breites Absorptionsmaximum bei $246 \mathrm{~nm}$.

Die Versuche mit den Rattenleberhomogenaten ergaben folgendes: Nach Zusatz von Clofedanol-Base ließen sich nach Extraktion nur zwei basische Verbindungen nachweisen. Durch den dünnschichtchromatographischen Vergleich sowie durch den Vergleich der UV-, IR- und Massenspektren mit denen des authentischen Materials wurden sie als Clofedanol-Base und Metabolit VI identifiziert.

Bei den Versuchen mit Metabolit VI wurde in dem sauren Auszug Metabolit V dünnschichtchromatographisch und massenspektrometrisch nachgewiesen. Der alkalische Auszug enthielt nur unveränderte Ausgangssubstanz.

Bei der Untersuchung des Harnes auf Aminosäuren wurde eine Substanz gefunden (Metabolit VII), die in dem medikamentenfreien Urin nicht nachgewiesen wurde. Die Struktur von VII als N,N-Dimethylaminoessigsäure ergab sich durch Vergleich des IR-Spektrums mit dem von authentischem Material und durch die Identität des aus VII mit Diazomethan erhaltenen Produktes mit dem handelsüblichen Betain (Schichtchromatographie und IR-Spektrum).

Nach den vorliegenden Untersuchungen wird Clofedanol auf verschiedenen Wegen metabolisiert, z.T. jedoch unverändert ausgeschieden. Durch Konjugation mit Glucuronsäure entsteht Clofedanol-Glucuronid (Metabolit VIII) und durch Wasserabspaltung wird 1-(oChlorphenyl)-1-phenyl-3-dimethylaminopropen-(1) gebildet, welches durch oxidative Desaminierung in $\beta$-(o-Chlorphenyl)- $\beta$-phenylacrolein (Metabolit V) umgewandelt wird. Eine $\beta$-oxidative Spaltung von Metabolit $\mathrm{V}$ würde die Entstehung von $o$-Chlorbenzophenon (Metabolit III) erklären (8). Es ist allerdings nicht auszuschließen, daß ein Teil von Metabolit V zuerst dehalogeniert wird und dann durch $\beta$-Oxidation in Benzophenon (Metabolit II) umgewandelt wird. Da halogenfreie Metaboliten nur in der letzten Oxidationsstufe des Clofedanol gefunden wurden, wird man nicht ausschließen können, daß Benzophenon auch durch Dehalogenierung von $o$-Chlorbenzophenon entstanden sein kann. Nach den bisherigen Erfahrungen (9) ist anzunehmen, daß Benzhydrol (Metabolit IV) durch Reduktion von Benzophenon entstanden ist.

Unklar ist, auf welchem Wege $o$-(Chlorphenyl)-phenylmethan (Metabolit I) und N,N-Dimethylaminoessigsäure (Metabolit VII) gebildet werden.

\section{Danksagung}

Dem Verband der Chemischen Industrie e.V., Fonds der Chemischen Industrie, danken wir für die gewährte Unterstützung. 


\section{Literatur}

1. Eckhardt, G., Goenechea, S. \& Gielsdorf, W. (1977), dieses J. $15,115-120$.

2. Stahl, E. (1967), Dünnschichtchromatographie. Ein Laboratoriumshandbuch. Springer Verlag, Berlin-Heidelberg-New York.

3. Merck, E. (1972), Anfärbereagenzien für Dünnschicht- und Papierchromatographie, Darmstadt.

4. Awapara, J. (1948), Arch. Biochem. 19, 172-173.

5. Ronayne, J., Williams, D. H. \& Bowie, J. H. (1966), J. Am. Chem. Soc. $88,4980-4984$.

6. Cooks, R. G. (1969), Org. Mass. Spectrom. 2, 481-519.

7. Kuschel, H. \& Grützmacher, H. F. (1974), Org. Mass. Spectrom. 9, 395-402, 403-407 und 408-417.

8. Karlson, P. (1974), Kurzes Lehrbuch der Biochemie. 9. Aufl. Georg-Thieme-Verlag, Stuttgart.

9. Ritschèl, W. A. (1973), Angewandte Biōphärmazie. Wissenschaftliche Verlagsgesellschaft m.b.H., Stuttgart.

Prof. Dr. S. Goenechea Institut für Gerichtl. Medizin der Univễésität Bonn Stiftsplatz 12, 5300 Bọn 